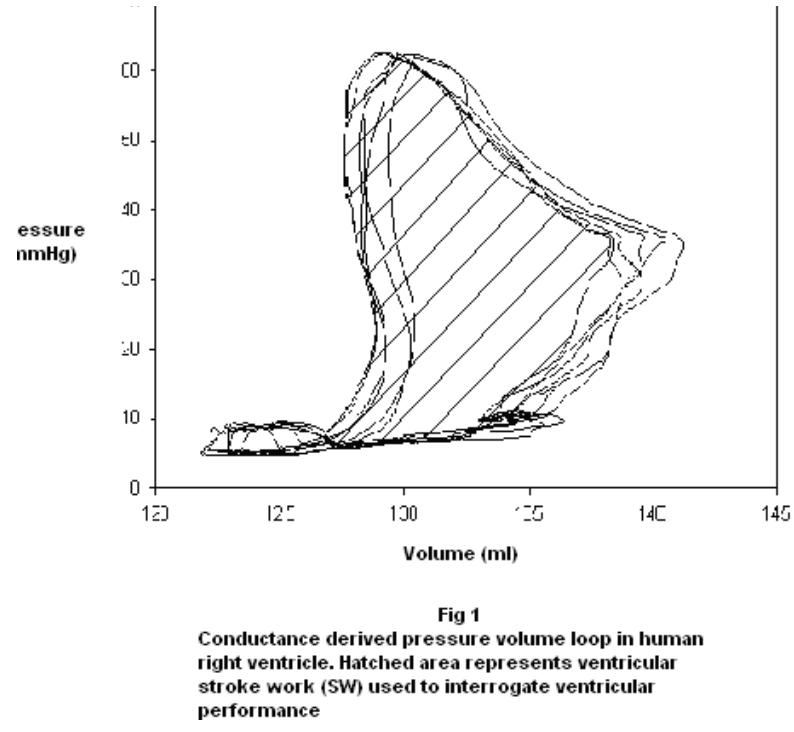

Abstract S41 Figure 1

Methods We have followed the Oxford cohort of 189 patients for two years to assess real vascular event rates and the incidence of accidents and hospital admissions. The events have been verified by inspection of hospital notes and phone calls to patients. The CPAP/ No CPAP allocation was based on intention to treat. At the end of the original 6 month trial, $71 \%$ of patients, initially randomised to CPAP, stated an intention to continue CPAP.

Results The table shows the 2 year incidence of accidents at home, work and driving (66/189), the incidence of new vascular events (25/189), and all cause mortality (4/189).

Conclusions The event rates are moderate, and there is no statistically significant difference between the groups. A power calculation, based on these figures, suggests that in order not to miss a $30 \%$ reduction in relative risk over 2 years ( $4 \%$ absolute reduction), with $90 \%$ power, would require 2,540 patients randomised; for accidents, 880 ; for mortality over 6,000 .

Abstract S42 Table 1 Summary of the 2 year accident and vascular event incidence in Oxford MOSAIC patients

\begin{tabular}{llcrr}
\hline & \multicolumn{1}{c}{ Where } & No CPAP & CPAP & Total \\
\hline \multirow{2}{*}{ Accidents } & Home & 22 & 25 & 47 \\
& Work & 4 & 5 & 9 \\
& Driving & 6 & 4 & 10 \\
\multirow{4}{*}{ Vascular events } & Total & 32 & 34 & 66 \\
& Cardiovascular death & 2 & 1 & 3 \\
& Other events & 15 & 7 & 22 \\
All cause mortality & Total & 17 & 8 & 25 \\
\hline
\end{tabular}

\section{S43 FRACTAL ANALYSIS OF WHOLE BLOOD INCIPIENT CLOT CONFIRMS AN EARLY MORNING PROTHROMBOTIC STATE IN PATIENTS WITH UNTREATED OBSTRUCTIVE SLEEP APNOEA SYNDROME}

doi:10.1136/thoraxjnl-2012-202678.049

${ }^{1} \mathrm{M}$ Wilczynska, ${ }^{1} \mathrm{~K}$ Lewis, ${ }^{2} \mathrm{M}$ Lawrence, ${ }^{2} \mathrm{~S}$ Stanford, L Nicolle', 2 PA Evans. 'Prince Philip Hospital, Swansea, UK; '2Morriston Hospital, Swansea, UK

Introduction Obstructive sleep apnoea syndrome (OSAS) independently increases cardiovascular risk and a pro-thrombotic state has been at least partly implicated. Using fractal analysis we have developed a new biomarker called fractal dimension (Df) to assess the microstructure of incipient clot in whole blood. Df relates to the kinetics of clot formation and quantifies clot fibrin network microstructure as it forms. A higher $\boldsymbol{D} \boldsymbol{f}$ represents a more pro-coaguable state. Healthy volunteers have a reproducible $\boldsymbol{D} \boldsymbol{f}$ of $1.74 \pm 0.07$. (1)

Aim To see if $\boldsymbol{D} \boldsymbol{f}$ changes after sleep in OSAS and symptomatic snorers.

Methods 28 patients with newly diagnosed and untreated OSAS: 24 males, mean $+\mathrm{SD} B M I=38 \pm 7.3 \mathrm{~kg} / \mathrm{m}^{2}$, age $56.8 \pm 10.7$ years, $4 \%$ Dip-rate $(D R)=47.8 \pm 32.9$ events/hour, Epworth Sleepiness Score (ESS)13.1 \pm 5.0 .17 symptomatic Controls with symptoms of OSAS but who had negative sleep studies: 14 males, BMI $=32.1 \pm 6.8 \mathrm{~kg} / \mathrm{m}^{2}$, age $52.7 \pm 13.6$ years, $4 \% \mathrm{DR}=5.6 \pm 3.2$ events/hour, ESS $11.3 \pm 6.6$. We excluded anyone on warfarin, heparin or with a family history of bleeding, thromboses. $36 \%$ in the OSAS group and $24 \%$ in the Controls were prescribed aspirin but there were no changes in medications throughout the study. Blood was collected at $4 \mathrm{pm}$ then $8 \mathrm{am}$ the following morning, immediately after an inpatient limited channel overnight sleep study (Visilab, Stowood Instruments, UK). Samples were tested for fractal analysis (AR-G2 Rheometer, TA Instruments, UK) and routine APTT, PT, fibrinogen levels, platelet aggregation. NCT01525160.

Results $\boldsymbol{D} \boldsymbol{f}$ was significantly higher in the early am (post sleep) $(p<0.001)$ than in the afternoon in OSAS but there was no diurnal variation in the Control group $(p=N S)$ Figure 1 . There were no statistically significant differences for routine coagulation and platelet function tests both within and between groups.

Conclusion OSAS is associated with a significantly increased prothrombotic state in the morning that is only detected by $\boldsymbol{D f}$. Larger numbers will allow sub-group analyses (e.g. severity of OSAS, aspirin effects) and seeing possible response to CPAP therapy. This preliminary data suggest $\boldsymbol{D} \boldsymbol{f}$ could be used as a new sensitive biomarker to assess vascular risk.

\section{Reference}

1. Evans PA et al. Blood. 2010; 116:3341-3346.

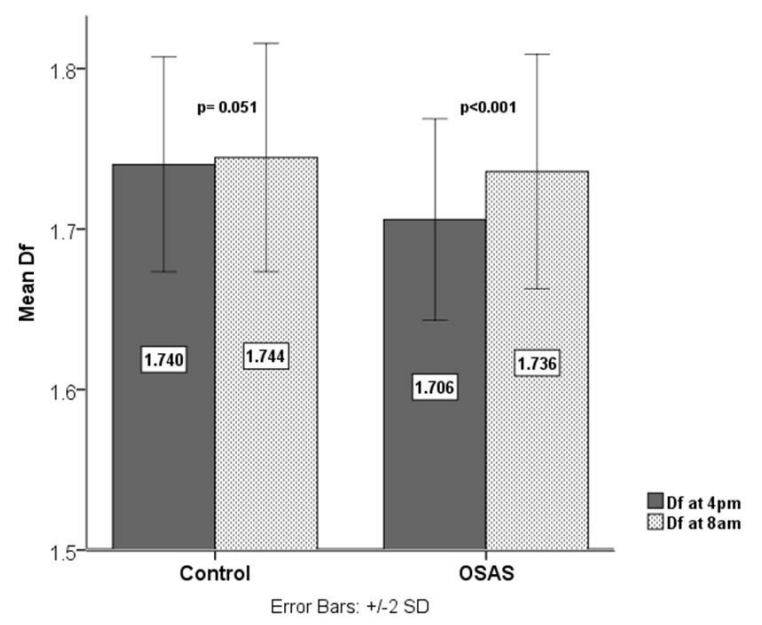

Abstract S43 Figure 1

\section{S44 7 DAYS ACTIGRAPHY MONITORING IS EQUIVALENT TO 14 DAYS ACTIGRAPHY MONITORING IN PATIENTS WITH SLEEP DISORDERS}

doi:10.1136/thoraxjnl-2012-202678.050

'S Briscoe, ${ }^{2} \mathrm{E}$ Hardy, ${ }^{1} \mathrm{C}$ Kosky, 'A Williams, 'N Hart, J Steier. 'Guy's and St Thomas' NHS Foundation Trust, London, UK; ${ }^{2}$ King's College London, London, UK

Introduction Actigraphy is a valid measure to assess sleep and circadian rhythm abnormalities. It is listed in the diagnostic criteria 